\title{
Arthroscopic revision ankle arthrodesis with fluoroscopic guidance: two cases
}

\author{
ELLY TREPMAN* AND JOHN E. FEMINO† \\ *Section of Orthopaedic Surgery, Department of Surgery, University of Manitoba, Winnipeg, \\ Canada and $\dagger$ Department of Orthopaedic Surgery, University of Michigan, Ann Arbor, USA
}

\section{Summary}

Two patients who had non-union of a previous open ankle arthrodesis were treated with revision arthrodesis using arthroscopic debridement and percutaneous cannulated screw fixation. The first patient had no signs of infection on preoperative aspiration and intraoperative cultures of the non-union; the second patient had had lateral wound breakdown and osteomyelitis after the primary arthrodesis procedure, which resolved after debridements, intravenous antibiotics and musculocutaneous free flap coverage. In both cases, arthroscopic debridement of the non-union was facilitated with fluoroscopic guidance for initial placement of instruments. Cancellous bone graft from the proximal tibia was placed into the non-union site with cannula and trocar through the arthroscopic portals, and tibiotalar fixation was achieved with cannulated $7.3 \mathrm{~mm}$ screws. Postoperative electrical bone stimulation was used. In the first patient, union was complete 12 weeks after surgery; in the second patient, who continued to smoke against advice, consolidation of some areas in the tibiotalar space was noted at 6.5 months after surgery and he eventually resumed high demand work. These cases demonstrate that arthroscopic ankle arthrodesis may be used successfully for revision of non-union, with potentially lower risk of wound complications compared with open methods.

Keywords: arthroscopy; treatment; non-union

\section{Introduction}

Ankle arthrodesis is a useful procedure for the management of mechanical pain in the arthritic ankle [1]. Open arthrodesis may be an extensive procedure involving fibulectomy [1, 2], medial malleolar excision [1] and bone grafting. The procedure may cause marked postoperative pain and the major soft tissue dissection required may contribute to the high rate of complications, which may include infection, wound slough, non-union and amputation [3]. Revision arthrodesis for non-union may have a greater risk of complications because of disruption of the soft tissues from previous surgery [4].

In recent years, advances in arthroscopic methods of joint debridement [5, 6] and cannulated screw fixation have enabled the development of arthroscopically assisted primary ankle arthrodesis [5, 7-15]. Advantages of this method include the use of small incisions for arthroscopic debridement and percutaneous screw placement, which may reduce the potential for wound slough and infection, decrease postoperative pain, and increase the rate of fusion because of less disruption of osseous blood 
supply, obviating the necessity of a bone graft. Furthermore, absence of fibulectomy or large bone cuts on the tibia and talus may facilitate alignment of the talus to the tibia, thereby reducing the risk of malunion [14, 15].

However, disadvantages of arthroscopically assisted ankle arthrodesis may include limitations in correcting deformity [9-11, 13] and the potential technical difficulty of the procedure [16]. Furthermore, the procedure has not been used for revision of non-union, for which the technical difficulties of debridement, bone loss and alignment may be greater, but in which potential benefits of decreased wound dissection may be especially important because of previous surgical disruption of the soft tissues.

In this report, the successful use of arthroscopic debridement and percutaneous screw fixation in revision ankle arthrodesis for non-union is described.

\section{Materials and methods}

Two patients presented with non-union of previous ankle arthrodesis.

\section{Case 1}

A 59-year-old woman had sustained an acute ankle fracture 14 years earlier and, despite initial treatment with open reduction, she developed posttraumatic ankle arthritis. In January 1997 she was treated elsewhere with an open ankle arthrodesis, including fibular osteotomy, sparing of the lateral malleolus, tibial and talar flat cut osteotomies, and internal screw fixation. Non-union of the arthrodesis persisted despite electrical bone stimulation. She had quit smoking 15 years earlier.

On presentation 14 months after the primary open arthrodesis, she noted pain across the anterior ankle, associated with walking, decreased with a removable cast boot (PneumaticWalker, Aircast, Summit, NJ, USA). Examination showed well-healed scars at the medial and lateral malleoli, plantigrade foot alignment and limited ankle motion associated with a painless 'clicking' sensation. Radiographs showed radiolucency at the tibiotalar, fibulotalar and tibiofibular regions, and radiolucency about fixation screws (Figure 1). Computerized tomography (CT) scan confirmed non-union. The white blood cell count was 9400 cells per $\mathrm{mm}^{3}$, with no left shift. The erythrocyte sedimentation rate (ESR) was $48 \mathrm{~mm} / \mathrm{h}$. Aspirate of the non-union had no cells or bacteria on gram stain, and culture was negative.

The patient had minimal pain with a custommoulded leather ankle brace with built-in plastic ankle foot orthosis, but continued to have anterior ankle pain. Reexamination showed anterior ankle pain with dorsiflexion and plantarflexion of the ankle region and tenderness at the anterior ankle joint. The patient elected to have revision ankle arthrodesis 16 months after primary open arthrodesis.

\section{Case 2}

A 39-year-old-man had sustained multiple left lower extremity injuries in a motor vehicle accident 17 years earlier, including left ankle and hip fracture-dislocations. A total hip arthroplasty 7 years earlier was complicated with deep infection, which was treated with implant removal, intravenous antibiotics and revision total hip arthroplasty 5 years earlier. Left ankle post-traumatic arthritis was treated elsewhere with open ankle arthrodesis 2 months before presentation, including distal fibulectomy, medial malleolar osteotomy, and internal fixation with screws, staples and a calcaneotalotibial pin which was removed 2 weeks after surgery. Subsequently, the lateral wound developed a 1 inch length of skin necrosis with drainage, and the patient was treated with hospital admission, debridement, removal of staples, external fixation and antibiotics. The operative cultures grew Enterobacter cloacae and Pseudomonas aeruginosa, and he was treated with ciprofloxacin and vancomycin. However, he developed fever to $103.5^{\circ} \mathrm{F}$; the white blood cell count was 5600 cells per $\mathrm{mm}^{3}$ and the ESR was $69 \mathrm{~mm} / \mathrm{h}$.

Upon transfer to this service, examination showed a lateral ankle wound (diameter, 1 inch; depth, 1 inch) with no purulence, and there was serous drainage from two of the external fixator pin sites. An indium-labelled white blood cell scan showed increased activity in the ankle and hindfoot region, and CT scan showed non-union of the ankle arthrodesis.

At initial open debridement, necrotic soft tissue and bone was excised from the wound margins, 

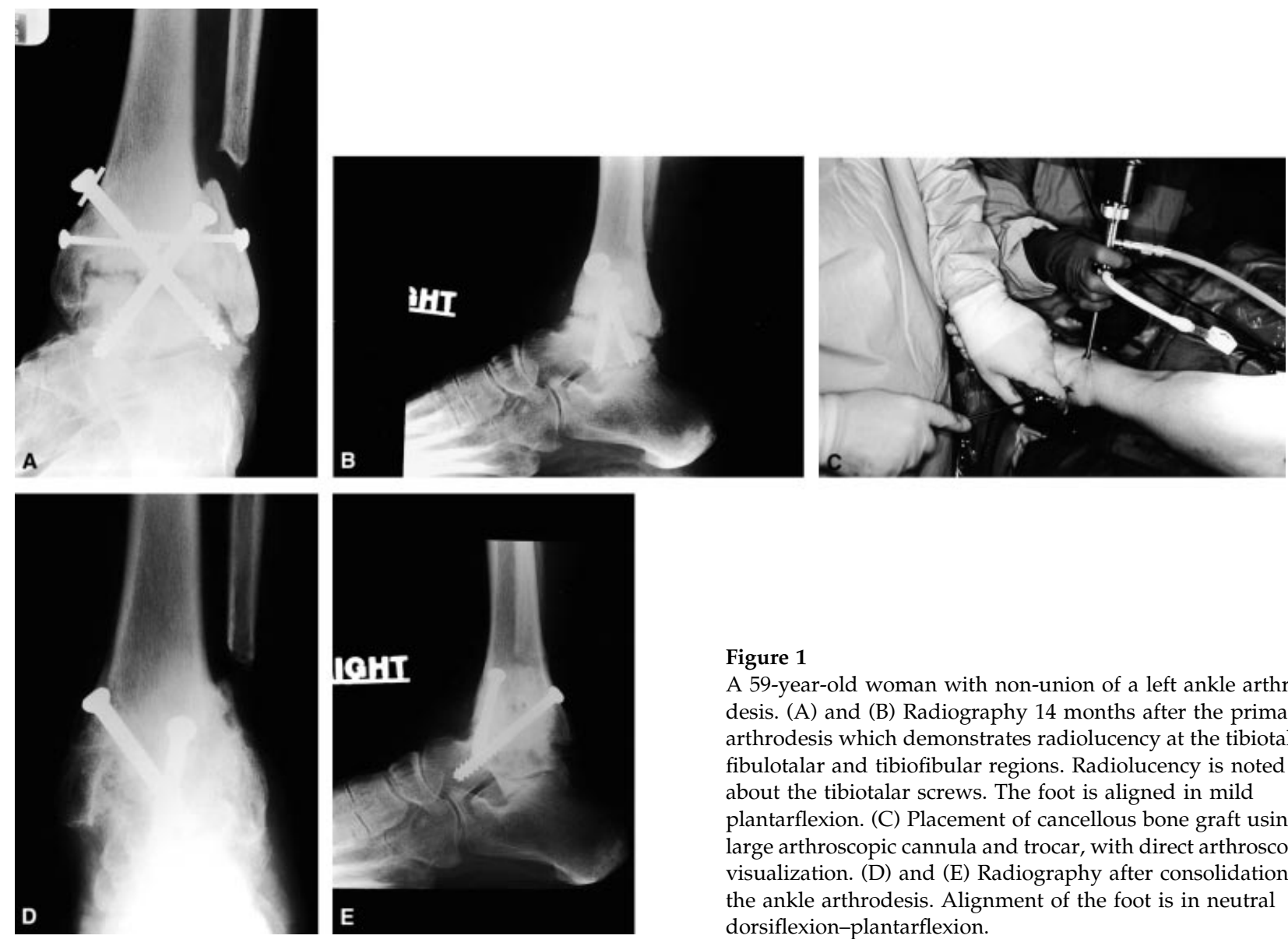

Figure 1

A 59-year-old woman with non-union of a left ankle arthrodesis. (A) and (B) Radiography 14 months after the primary arthrodesis which demonstrates radiolucency at the tibiotalar, fibulotalar and tibiofibular regions. Radiolucency is noted about the tibiotalar screws. The foot is aligned in mild plantarflexion. (C) Placement of cancellous bone graft using a large arthroscopic cannula and trocar, with direct arthroscopic visualization. (D) and (E) Radiography after consolidation of the ankle arthrodesis. Alignment of the foot is in neutral dorsiflexion-plantarflexion.

subcutaneous tissues and ankle non-union site. The wound extended to the subtalar joint, which contained necrotic articular cartilage and granulation tissue. After this extensive debridement, treatment included repeat debridement 3 days later and lateral ankle musculocutaneous free flap coverage, which healed well. The intravenous antibiotics were discontinued after a 7-week course. Ten days later the patient was readmitted because of a pin track infection, which rapidly resolved with removal of the external fixator, curettage of the pin sites and intravenous antibiotics for several days followed by oral antibiotics for 2 weeks.

The ankle was immobilized in a removable U-splint, and he continued with non-weightbearing and external electrical bone stimulation. However, follow-up magnetic resonance imaging (MRI) 4 months after the primary arthrodesis showed tibiotalar non-union; marrow oedema in the talus, calcaneus and distal tibia; and subtalar joint degen- erative changes. He progressed to full-weightbearing in a removable cast boot with a heel pad. Repeat white blood cell count was 7000 cells per $\mathrm{mm}^{3}$ and the ESR was $2 \mathrm{~mm} / \mathrm{h}$. With persistence of the nonunion (Figure 2), the patient elected to have revision ankle arthrodesis 5.5 months after primary open arthrodesis.

\section{Surgical technique}

Revision ankle arthrodesis was done under general anaesthesia, with a proximal thigh tourniquet. The patients were placed in the supine position on the operating table with a flat radiography leg extension board, and had a blanket roll placed behind the ipsilateral greater trochanter to maintain neutral lower extremity rotation. Sterile preparation included the foot to the distal thigh to facilitate determination of correct foot rotation and enable harvesting of proximal tibial bone graft. 

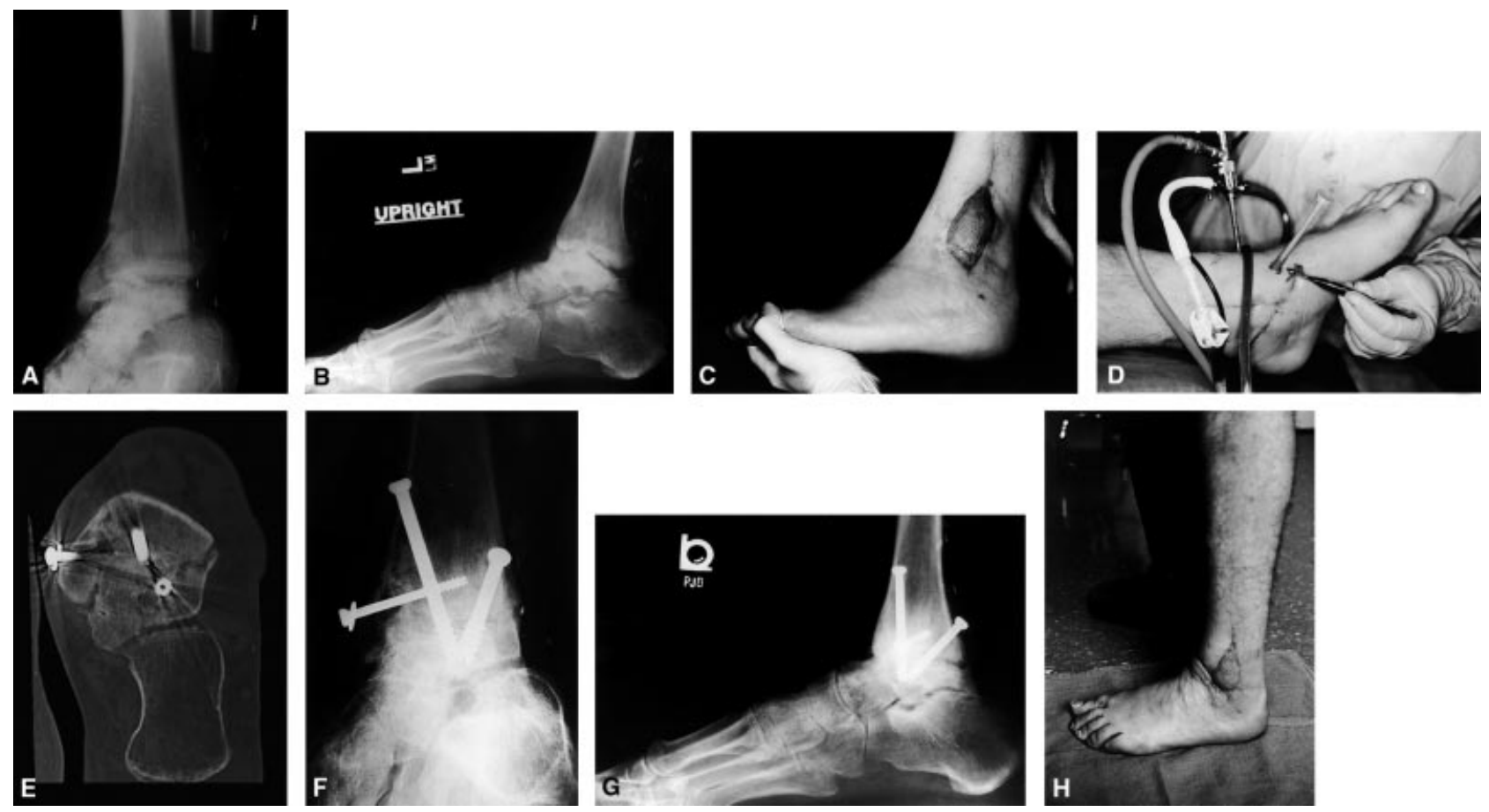

Figure 2

A 39-year-old man with non-union of a left ankle arthrodesis (A) and (B) Radiography after debridement, intravenous antibiotics, muscle flap coverage and removal of external fixator. Radiolucency is noted at the tibiotalar region and at the site of previous medial malleolar osteotomy. Degenerative changes are also noted at the subtalar joint. The foot is aligned in mild plantarflexion. (C) Preoperative appearance of the lateral ankle showing the healed free muscle flap graft and alignment in mild plantarflexion. (D) Placement of cancellous bone graft using a plastic cannula and trocar, with direct arthroscopic visualization. (E) CT scan 6.5 months after revision surgery showing areas of tibiotalar fusion and some areas of persistent radiolucency. Degenerative changes of the subtalar joint are noted. (F) and (G) Radiography at 13 months after arthroscopic revision ankle arthrodesis. Solid union is noted at the central and anterior tibiotalar region. (H) Postoperative clinical alignment of the foot in neutral dorsiflexion-plantarflexion.

For arthroscopic debridement, accurate portal placement was imperative because of the absence of a joint space or mobility, which would cause difficulty in placing debriding instruments into and across the non-union. Therefore, prior to commencing surgery, lateral fluoroscopic radiography, with a straight surgical instrument at the anterior ankle, was done to determine accurate position of the arthroscopic portals directly anterior to, and in the plane of, the non-union. After making the anterolateral and anteromedial arthroscopic portals with skin incision and subcutaneous spreading, curettes were accurately placed into the non-union site with direct fluoroscopic guidance, and initial fluoroscopically guided curettage was done to open up the nonunion and enable subsequent placement of the arthroscopic instruments. In case 1, retained screws were removed with fluoroscopic guidance; in case 2, there was no retained hardware.
Subsequently, arthroscopic debridement was done with direct visualization using a $4.0-\mathrm{mm}, 30^{\circ}$ arthroscope $[7,10,13]$ connected to gravity saline inflow and videocamera; an accessory third portal was not required. Debridement was initially performed with straight and angled curettes, and then with a motorized $4.5 \mathrm{~mm}$ shaver and $5.5 \mathrm{~mm}$ burr, interchanging the arthroscope and debriding instruments between medial and lateral portals [9-11, 13]. The surfaces of the talar dome, tibial plafond, medial and lateral talar walls, and malleoli were debrided. With progressive debridement from anterior to posterior, the non-union space was widened adequately to enable complete debridement [13], facilitated with plantarflexion of the foot and manual distraction alone [12]; neither external weight strap [7,9] nor external fixator was required. Debridement of fibrous tissue and dense sclerotic bone was done until healthy-appearing cancellous bony surfaces 
were exposed throughout the ankle, and multiple small drill holes were made on the talar dome and tibial plafond; bleeding from the cancellous surfaces was confirmed by direct arthroscopic visualization after deflation of the tourniquet (tourniquet time, $2.5 \mathrm{~h}$ in case 1 and $2.3 \mathrm{~h}$ in case 2), which then remained deflated for the duration of the procedure. In case 2, non-union of the medial malleolus osteotomy (Figure 2) was debrided percutaneously with curette and fluoroscopic guidance.

Cancellous bone graft was harvested from the proximal tibia with a modification of the previously described method $[17,18]$, by removing a rectangular anteromedial metaphyseal cortical window $(2 \mathrm{~cm}$ by $1 \mathrm{~cm}$ ) medial to the tibial tubercle, curetting out cancellous bone from the proximal tibia and replacing the cortical window. The cancellous bone graft was then placed into the arthrodesis site using a cannula and trocar $[5,19,20]$ through the arthroscopic portals with direct arthroscopic visualization (Figures 1C and 2D).

After removal of arthroscopic instruments, the foot and ankle was placed in the desired position of neutral dorsiflexion-plantarflexion, $0-5^{\circ}$ heel valgus position and $0-5^{\circ}$ foot external rotation. Plantarflexion deformity at the arthrodesis site was corrected by sequentially dorsiflexing the foot during arthroscopic debridement, with removal of anterior osteophytes and more anterior bone until neutral flexion had been achieved. Internal fixation was accomplished percutaneously with two cannulated $7.3 \mathrm{~mm}$ screws: one from posterior distal tibia to the talus and one from medial distal tibia to the talus. In case 2 , the medial malleolus non-union was fixed to the distal tibia with one $4.5 \mathrm{~mm}$ cannulated screw. Accurate placement of screws was facilitated by fluoroscopic evaluation with guide pins outside the ankle to determine direction and position, provisional fixation with percutaneously placed guide pins, and fluoroscopic confirmation of guide pin length and position. After placement of the screws, lateral, $\mathrm{AP}$, oblique and Broden fluoroscopic views were done to confirm that satisfactory bony alignment was achieved, screw threads completely crossed the arthrodesis site, and the screw tips were in the talus and not the subtalar joint $[10,11]$.

Postoperative management included external electrical bone stimulation (EBI Medical Systems, Parsippany, NJ, USA), non-weightbearing and immo- bilization with a well-padded plaster U-splint that was changed 1 day after surgery to a univalved fibreglass short leg cast. Sutures were removed after 2 weeks. The patients were advanced to touchdown weightbearing (case 1, removable walker boot [PneumaticWalker, Aircast, Summit, NJ, USA]; case 2, fibreglass short leg cast) after 4-5 weeks and full weightbearing after 8 weeks (removable walker boot). In case 1 , the removable walker boot was changed to a stirrup brace (Aircast Stirrup, Aircast) at 16 weeks, which was tapered to regular footwear at 21 weeks. In case 2, the removable walker boot was continued for 6 months and, subsequently, the patient used the removable walker boot and a moulded leather ankle brace with prolonged weightbearing.

\section{Results}

In both cases, operative deep tissue cultures at the time of arthroscopic debridement and revision arthrodesis were negative, and there were no clinical signs of active infection. In both cases, fibrous nonunion was present. In case 1, histological examination of debrided non-union tissue showed necrotic trabeculae, cartilaginous changes, medullary fibrosis, and fragments of degenerative fibrocartilage and articular cartilage. In case 2, the previous deep infection was clinically resolved, and histological examination of debrided non-union tissue showed remodelling bone, acellular trabeculae, medullary fibrosis and rare plasma cells.

In both cases, postoperative radiographs showed maintenance of alignment and position of internal fixation hardware. In case 1, progressive radiographic consolidation was complete at 12 weeks after revision surgery; at 6 months and 9 months after revision surgery, the patient was walking pain-free with normal flat shoes (Figure 1). In this patient, AOFAS Ankle-Hindfoot Score (maximum 92 points for ankle arthrodesis) [21] improved from 8 points before surgery to 77 points at follow-up evaluation.

In case 2, radiography showed progressive consolidation but persistence of radiolucency. The patient continued to smoke cigarettes despite being advised of the documented association between smoking and non-union [22]; he eventually quit smoking 5 months after revision surgery. CT scan 6.5 months after revision surgery showed areas of 
tibiotalar fusion, with persistent areas of non-union anteriorly and at the medial malleolus (Figure 2E); degenerative changes of the subtalar joint were noted. Eight months after revision surgery, he noted mild, occasional pain at the medial malleolus and medial and lateral subtalar region, but no pain when using a removable walker boot. At 13 months after revision surgery, he had returned to high-demand work directing an asphalt pavement company, $14 \mathrm{~h}$ per day on his feet, against advice, using a heavy work boot worn inside a removable walker boot; he used a moulded leather ankle brace when not at work. He noted some pain at the medial subtalar and dorsal Chopart areas, but deferred on further surgery. Radiography at 13 months after revision surgery showed consolidation of the ankle arthrodesis centrally and anteriorly, with some residual posterior radiolucency, and consolidation of the medial malleolus (Figures 2F and G). In this patient, AOFAS Ankle-Hindfoot Score [21] improved from 5 points before surgery to 58 points at follow-up evaluation.

\section{Discussion}

The cases demonstrate that arthroscopic ankle arthrodesis may be successfully applied to nonunions of previous open ankle arthrodesis, even with history of previous infection, free muscle flap coverage and bone loss. Previous studies had used this method for primary arthrodesis of arthritic ankle joints [7, 9-13, 19, 23] and the current report extends the indications of this procedure to more complex revision cases.

The technical difficulties of performing arthroscopic revision ankle arthrodesis included those of primary arthroscopic ankle arthrodesis, such as equipment demands, longer operating time and laborious effort in performing a thorough arthroscopic debridement [7]. The revision procedures were more difficult than primary arthrodesis because of the presence of distorted bony anatomy and dense scar tissue at the non-union site. The presence of normal bony contours, which may facilitate primary arthroscopic ankle arthrodesis [9], may be absent if the primary case was done with techniques involving bone excision such as tibiotalar flat-cut or chevron resection and fibulectomy [1] (Figures 1 and 2). Previous experience with ankle arthroscopy and primary arthroscopic ankle arthrodesis facilitated the performance of the revision procedure [11].

The use of fluoroscopic guidance in the initial percutaneous placement of the curettes facilitated the opening of the non-union site, enabled accurate placement of the straight instruments all the way from the front to the back of the ankle non-union plane, and decreased the risk of erroneous placement of arthroscopic instruments in normal bone outside the non-union ('false pass'). The use of curettes to open the non-union site enabled the procedure to be accomplished with manual distraction alone [11, 12], and neither external fixator [7, 10, $13,19]$ nor external weight strap $[7,9]$ was required. Fluoroscopic confirmation of depth of curette position at the end of debridement was available to confirm that complete debridement to the posterior part of the ankle had been accomplished. Furthermore, no posterior portal was used in these cases [9], and sufficient visualization was achieved with gravity inflow of fluid [10], which may potentially reduce the risk of pressurization of fluid out the nonunion site into the compartments of the leg or foot if a fluid pump is used.

The potential advantages of the arthroscopic method for revision of ankle non-union included the marked reduction in soft tissue dissection compared with open arthrodesis [5, 7, 9, 11-13, 15]. This is a major potential advantage in revision cases with soft tissues at high risk for wound problems [12] such as the patient with a previous free flap coverage [9, 24], extensive soft-tissue scarring, previous deep infection or venous stasis disease. Additional advantages of the arthroscopic method included the lower levels of postoperative pain than with open arthrodesis [11, 13, 14] and the apparently faster fusion rate $[9,13]$. Furthermore, the use of bone graft from the proximal tibia $[17,18]$ obviated the potential morbidity of an iliac crest bone graft $[25,26]$, and the use of a cannula enabled the percutaneous delivery of the bone graft $[5,19,20]$ without the necessity of an open procedure [13].

In both cases described, preoperative alignment was neutral varus/valgus position, but both cases had plantarflexion deformity which was able to be corrected. The presence of greater deformity may make it more difficult to achieve the desired alignment [7]. However, complete bone-to-bone contact 
might not be required with an arthroscopic method [11] in which cancellous bone is exposed with extensive burring, and the use of a cancellous bone graft may be helpful. In one case (Figure 2), an apparent fibrous union was, on follow-up radiography and CT scanning, shown to represent a partial bony union which, as shown with some fibrous unions following primary ankle arthrodesis [19], may provide satisfactory pain relief and functional result.

\section{References}

1 Mann RA. Arthrodesis of the foot and ankle. In: Mann RA, Coughlin MJ, Eds., Surgery of the Foot and Ankle, Sixth Edition, Chapter 15. St Louis: Mosby, 1993: 673-713.

2 Wang G-J, Shen W-J, McLaughlin RE et al. Transfibular compression arthrodesis of the ankle joint. Clin Orthop Rel Res 1993; 289: 223-227.

3 Helm R. The results of ankle arthrodesis. J Bone Joint Surg 1990; 72-B: 141-143.

4 Kirkpatrick JS, Goldner JL, Goldner RD. Revision arthrodesis for tibiotalar pseudarthrosis with fibular onlay-inlay graft and internal screw fixation. Clin Orthop Rel Res 1991; 268: 29-36.

5 Ewing JW, Tasto JA, Tippett JW. Arthroscopic surgery of the ankle. AAOS Instr Course Lect 1995; 44: 325-340.

6 Parisien JS. Arthroscopic surgery of the ankle. In: Parisien JS Ed. Arthroscopic Surgery, Chapter 22. New York: McGraw-Hill, 1988: 259-281.

7 Glick JM, Morgan CD, Myerson MS et al. Ankle arthrodesis using an arthroscopic method: long-term follow-up of 34 cases. Arthroscopy 1996; 12: 428-434.

8 Glick JM, Ferkel RD. Arthroscopic ankle arthrodesis. In: Ferkel RD, Ed., Arthroscopic Surgery: the Foot and Ankle, Chapter 11. Philadelphia: Lippincott-Raven, 1996: 215-229.

9 Corso SJ, Zimmer TJ. Technique and clinical evaluation of arthroscopic ankle arthrodesis. Arthroscopy 1995; 11: 585-590.

10 Dent CM, Patil M, Fairclough JA. Arthroscopic ankle arthrodesis. J Bone Joint Surg 1993; 75-B: 830-832.

11 Ogilvie-Harris DJ, Lieberman I, Fitsialos D. Arthroscopically assisted arthrodesis for osteoarthrotic ankles. J Bone Joint Surg 1993; 75-A: 1167-1174.
12 Jerosch J, Steinbeck J, Schröder M et al. Arthroscopically assisted arthrodesis of the ankle joint. Arch Orthop Trauma Surg 1996; 115: 182-189.

13 Myerson MS, Quill G. Ankle arthrodesis. A comparison of an arthroscopic and an open method of treatment. Clin Orthop Rel Res 1991; 268: 84-95.

14 Fitzgibbons TC. Arthroscopic ankle debridement and fusion: indications, techniques, and results. AAOS Instr Course Lect 1999; 48: 243-248.

15 O'Brien TS, Hart TS, Shereff MJ et al. Open versus arthroscopic ankle arthrodesis: a comparative study. Foot Ankle Int 1999; 20: 368-374.

16 Paremain GD, Miller SD, Myerson MS. Ankle arthrodesis: results after the miniarthrotomy technique. Foot Ankle Int 1996; 17: $247-252$.

17 O'Keeffe RM, Riemer BL, Butterfield SL. Harvesting of autogenous cancellous bone graft from the proximal tibial metaphysis. A review of 230 cases. J Orthop Trauma 1991; 5: 469-474.

18 Catone GA, Reimer BL, McNeir D et al. Tibial autogenous cancellous bone as an alternative donor site in maxillofacial surgery: a preliminary report. J Oral Maxillofac Surg 1992; 50: 1258-1263.

19 Crosby LA, Yee TC, Formanek TS et al. Complications following arthroscopic ankle arthrodesis. Foot Ankle Int 1996; 17: 340-342.

20 Waldron VD. Delivering bone grafts percutaneously. Am J Orthop 1999; 28: 195.

21 Kitaoka HB, Alexander IJ, Adelaar RS et al. Clinical rating systems for the ankle-hindfoot, midfoot, hallux, and lesser toes. Foot Ankle Int 1994; 15: 349-353.

22 Cobb TK, Gabrielsen TA, Campbell DC et al. Cigarette smoking and nonunion after ankle arthrodesis. Foot Ankle 1994; 15: 64-67.

23 Turan I, Wredmark T, Felländer-Tsai L. Arthroscopic ankle arthrodesis in rheumatoid arthritis. Clin Orthop Rel Res 1995; 320: $110-114$

24 Morgan CD. Arthroscopic ankle arthrodesis. In: Guhl JF, Ed., Foot and Ankle Arthroscopy, Chapter 14. Thorofare, New Jersey: Slack, 1993: 161-169.

25 Arrington ED, Smith WJ, Chambers HG et al. Complications of iliac crest bone graft harvesting. Clin Orthop Rel Res 1996; 329: 300-309.

26 Goulet JA, Senunas LE, DeSilva GL et al. Autogenous iliac crest bone graft. Complications and functional assessment. Clin Orthop Rel Res 1997; 339: 76-81. 